\title{
Ethanol Inhibition of a Bacterium (Acinetobacter calcoaceticus) in Chemostat Culture
}

\author{
By B. J. АВBOTT* \\ Corporate Research Laboratories, Esso Research and Engineering Company, \\ Linden, New Jersey, 07036, U.S.A.
}

(Received 27 September 1972)

\begin{abstract}
SUMMARY
Acinetobacter calcoaceticus, a soil isolate capable of utilizing ethanol as a sole carbon source, was cultivated in a chemostat under ethanol-limiting conditions. The pulse addition of ethanol (to less than $0.1 \%$ final concentration) to steady state cultures inhibited growth. Growth inhibition was accompanied by conversion of ethanol to acetate, after which growth resumed on the accumulated acetate and the culture returned to the original steady state. The final concentration of ethanol added and acetate accumulated were lower than levels that inhibited growth in batch culture. The rate of acetate synthesis after the pulse addition of ethanol was sufficient to support the maximum specific growth rate $\left(\mu_{\max }\right)$ although the growth rate immediately before ethanol addition was only $47 \%$ of $\mu_{\max }$. In batch culture acetate- or ethanol-grown cells preferentially consumed the acetate component of an ethanol-acetate mixture, but in chemostat cultures the presence of acetate did not prevent ethanol oxidation.
\end{abstract}

\section{INTRODUCTION}

Many micro-organisms can utilize ethanol as a primary source of carbon and energy. Unlike many carbon sources, ethanol in relatively low concentrations causes a reduction in growth rate. Hinshelwood (I952) found that the decrease in growth rate of batch cultures of Bacterium lactis aerogenes was a linear function of the ethanol concentration, as described by the following:

$$
\mu=\mu_{\mathrm{max}}{ }^{(1-a P)},
$$

where $\mu_{\max }=$ maximum specific growth rate, $P$ is the ethanol concentration and $a$ is an empirical constant. A similar result was obtained by Zines \& Rogers (I97I) utilizing turbidistat cultures of glucose-grown Aerobacter aerogenes. Earlier Zines \& Rogers (I970) in a study of the effect of pulse and step additions of ethanol to nitrogen-limited chemostat cultures showed that ethanol inhibition of $A$. aerogenes cultivated under such conditions was best described by a second or higher order equation.

The kinetics of ethanol inhibition also has been studied with ethanol-producing yeasts. Holzberg, Finn \& Steinkraus (1967) found that a modification of the Hinshelwood relationship described the inhibition of growth of batch cultures of Saccharomyces cerevisiae and that it was necessary to exceed a threshold concentration of $2.6 \%$ before inhibition occurred. In contrast, Aiba, Shoda \& Nagatani (I968) using continuous cultures found that there was an exponential decrease in growth rate of $S$. cerevisiae as the ethanol concentration increased. The relationship was expressed as

$$
\mu=\mu_{\mathrm{max}} \mathrm{e}^{-k}
$$

* Present address: Eli Lilly Co., Department MC 8I I, Indianapolis, Indiana, 46206, U.S.A. 
where $k$ is an empirical constant. Subsequently, Aiba \& Shoda (1969) reported that their data would also fit the following equation of non-competitive enzyme inhibition:

$$
\mu=\frac{\mu_{\max } k_{\mathrm{p}}}{k_{\mathrm{p}}+P}
$$

where $k_{\mathrm{p}}$ is the ethanol concentration that reduces the growth rate $50 \%$. Egamberdiev \& Jerusalimskii (1968) also observed this pattern of inhibition in batch cultures of Saccharomyces vini.

In the present study it was found that bacteria, grown in batch culture in the presence of ethanol, tolerated ethanol concentrations up to about $\mathrm{I} \cdot 0 \%$ before growth rate was reduced. However, chemostat cultures of ethanol-grown bacteria growing at less than $\mu_{\max }$ were inhibited at ethanol concentrations lower than $0.1 \%(w / v)$. Subsequent studies were conducted to establish the cause for this apparent increased sensitivity to ethanol.

\section{METHODS}

Organism. The organism used in these studies was a bacterium isolated from soil by ethanol enrichments. It was capable of utilizing ethanol as a sole carbon source. The organism was obligately aerobic, non-sporeforming, non-motile, usually appearing as pairs of coccoid cells. It was Gram-negative, but under some conditions tended to resist decolorization, and appeared to be 'Gram-variable'. Colonies were whitish to cream-coloured, sometimes appeared quite mucoid, and did not fluoresce. The organism was oxidase negative, catalase positive; it did not hydrolyse serum, casein, gelatin or starch nor produce $\mathrm{H}_{2} \mathrm{~S}$ or reduce nitrate. It grew on citrate, and produced acid from arabinose, xylose, galactose and glucose, but not from sucrose, fructose or glycerol. Its DNA contained about $4 \mathrm{I}$ mol \% GC. From these characteristics the organism was designated Acinetobacter calcoaceticus (Thornley, I967).

Cultivation. The medium for continuous cultivation comprised: $\mathrm{H}_{3} \mathrm{PO}_{4}, \mathrm{I} \cdot 5 \mathrm{~g} ; \mathrm{K}_{2} \mathrm{SO}_{4}$, $0.5 \mathrm{~g} ; \mathrm{MgSO}_{4} .7 \mathrm{H}_{2} \mathrm{O}, 0.25 \mathrm{~g} ; \mathrm{FeSO}_{4} .7 \mathrm{H}_{2} \mathrm{O}, 0.02 \mathrm{~g} ; \mathrm{MnSO}_{4} . \mathrm{H}_{2} \mathrm{O}, 0.02 \mathrm{~g} ; \mathrm{H}_{3} \mathrm{BO}_{4}, 0.5 \mathrm{mg}$; $\mathrm{CuSO}_{4} .5 \mathrm{H}_{2} \mathrm{O}, 0.4 \mathrm{mg} ; \mathrm{Na}_{2} \mathrm{MoO}_{2} .2 \mathrm{H}_{2} \mathrm{O}, 0.2 \mathrm{mg} ; \mathrm{ZnSO}_{4} .7 \mathrm{H}_{2} \mathrm{O}, 8.0 \mathrm{mg} ; \mathrm{CaCl}_{2}, 50.0 \mathrm{mg}$; $\mathrm{CoCl}_{2} .6 \mathrm{H}_{2} \mathrm{O}, 0 \cdot 2 \mathrm{mg}$; ethanol, $6 \cdot 0 \mathrm{~g}$; distilled $\mathrm{H}_{2} \mathrm{O}$, I 1 .

The medium was sterilized by filtration through a $0.22 \mu \mathrm{m}$ pore-size filter, stored in a glass holding vessel, and pumped into the fermenter as required. The $\mathrm{pH}$ in the fermenter was controlled at 7.0 by automatic additions of an ammonia solution. The 51 fermenter (Fermentation Design, Allentown, Pennsylvania, U.S.A.) contained 21 of medium and was maintained at $35^{\circ} \mathrm{C}$. Air was added at $\mathrm{I} \cdot 0$ vol./vol./min and the impeller operated at 700 rev./min. Dissolved oxygen was monitored by a galvanic probe (Fermentation Design) and the inlet air was enriched with oxygen, when necessary, to prevent an oxygen limitation. The $\mathrm{CO}_{2}$ content of the exit gas was measured with a $\mathrm{CO}_{2}$ analyser (Beckman Instrument Co., Fullerton, California, U.S.A.) and water vapour was removed with Aquasorb (Mallinckrodt, St Louis, Missouri, U.S.A.), a phosphorus pentoxide-based desiccant.

The medium used to cultivate Acinetobacter calcoaceticus in shake flasks was designated P-I and comprised: $\left(\mathrm{NH}_{4}\right)_{2} \mathrm{HPO}_{4}$, Io g; $\mathrm{K}_{2} \mathrm{HPO}_{4}, 5 \mathrm{~g} ; \mathrm{Na}_{2} \mathrm{SO}_{4}, 0.5 \mathrm{~g} ; \mathrm{MgSO}_{4} \cdot 7 \mathrm{H}_{2} \mathrm{O}, 0.4 \mathrm{~g}$; $\mathrm{FeSO}_{4} .7 \mathrm{H}_{2} \mathrm{O}, 0.02 \mathrm{~g} ; \mathrm{MnSO}_{4} .4 \mathrm{H}_{2} \mathrm{O}, 0.02 \mathrm{~g} ; \mathrm{NaCl}, 0.02 \mathrm{~g} ; \mathrm{H}_{3} \mathrm{BO}_{3}, 0.5 \mathrm{mg} ; \mathrm{CuSO}_{4} .5 \mathrm{H}_{2} \mathrm{O}, 0.04$ $\mathrm{mg} ; \mathrm{Na}_{2} \mathrm{MoO}_{4} \cdot 2 \mathrm{H}_{2} \mathrm{O}, 0.2 \mathrm{mg} ; \mathrm{ZnSO}_{4} \cdot 7 \mathrm{H}_{2} \mathrm{O}, 8 \cdot 0 \mathrm{mg} ; \mathrm{CaCl}_{2}, 50 \cdot 0 \mathrm{mg} ; \mathrm{CoCl}_{2} \cdot 6 \mathrm{H}_{2} \mathrm{O}, 0 \cdot 2 \mathrm{mg}$; distilled $\mathrm{H}_{2} \mathrm{O}$, I 1 . In shake-flask experiments ethanol was added at $0 . \mathrm{I} \%(\mathrm{w} / \mathrm{v})$ concentration and the medium was sterilized by filtration through a $0.22 \mu \mathrm{m}$ pore-size filter.

Measurements of growth rates and biomass concentration. Growth rates were measured in $300 \mathrm{ml}$ shake flasks with a cuvette side arm. Each flask received P-I medium $(50 \mathrm{ml})$ and 
was inoculated with bacteria growing exponentially in P-I medium ( $0 \cdot 1 \%$ ethanol) to an initial extinction $(E)$ between 0.05 and 0.10 , measured at $660 \mathrm{~nm}$ in a spectrophotometer (Spectronic 20, Bausch \& Lomb, Rochester, New York, U.S.A.). Measurements.of $E$ were made at time intervals during incubation of culture; each increase of $0 . \mathrm{I} E$ unit corresponded to an increase of $0.036 \mathrm{mg}$ dry biomass $\mathrm{ml}$. A linear relationship. between bacterial mass and $E$ was observed up to an $E$ of about 0.7 . Growth rate was expressed as the specific growth rate $\mu$, where

$$
\mu=\frac{\ln 2}{g}=\frac{\ln X-\ln X_{1}}{t_{2}-t_{1}}
$$

and $g$ is the mean generation time or doubling time; $X_{1}$ and $X_{2}$ are the biomass concentrations at time $t_{1}$ and $t_{2}$, respectively.

Analytical methods. Ethanol, acetate and acetaldehyde were detected by flame-ionization gas chromatography. The column was $6 \mathrm{ft} \times \frac{1}{4}$ inch stainless steel packed with 50/80 Porapak Q (Applied Science Laboratory, State College, Pennsylvania, U.S.A.). It was operated isothermally at $200{ }^{\circ} \mathrm{C}$ and the carrier gas flow was $30 \mathrm{ml}$ of helium $/ \mathrm{min}$. Samples were taken from shake flasks or fermenters and $5.0 \mu \mathrm{l}$ of the bacterial suspension were injected on to the column.

\section{RESULTS}

Continuous cultivation. A small amount of ethanol was added to an ethanol-limited steady state culture of Acinetobacter calcoaceticus growing at a specific growth rate of $0.33 \mathrm{~h}^{-1}$ (Fig. I). The concentration of ethanol in the chemostat immediately after the ethanol addition was $0.07 \%(\mathrm{w} / \mathrm{v})$. The addition of ethanol caused a rapid decline in both dissolved oxygen concentration and $\mathrm{CO}_{2}$ evolution, which persisted for $\mathrm{I} 8 \mathrm{~min}$. These declines were accompanied by the conversion of ethanol to acetate in the culture broth. After the ethanol was depleted acetate consumption began and $\mathrm{CO}_{2}$ evolution increased. Dissolved oxygen tension and $\mathrm{CO}_{2}$ evolution returned to their previous steady state values after the concentration of acetate returned to zero (Fig. I).

In other experiments similar changes in dissolved oxygen, $\mathrm{CO}_{2}$ evolution, ethanol and acetate concentrations occurred both at high and low biomass concentrations and at high and low growth rates, but the magnitude and duration of the changes varied. Rapid growth rates, high biomass concentrations and small additions of ethanol caused only short periods of non-steady state behaviour before the culture returned to steady state. When a large quantity of ethanol ( $\mathrm{I} \%$, w/v) was present a large amount of acetate accumulated, $\mathrm{CO}_{2}$ evolution declined for a prolonged period, and $\mu$ was reduced to such an extent that wash-out occurred.

The amount of acetate accumulated was dependent on the amount of ethanol added to ethanol-limited steady state cultures. In the experiment depicted in Fig. I the amount of acetate accumulated was $53.3 \mathrm{mmol}$, which approximately equalled the amount of ethanol added as a pulse addition plus the amount that entered the chemostat with the incoming medium during the period of acetate accumulation. Similar stoichiometries were found in other experiments where the growth rate and biomass concentration differed from those in Fig. I. In each experiment, between 85 and $100 \%$ of all the ethanol that entered the chemostat could be accounted for as accumulated acetate. These results indicated that after the pulse addition of ethanol, acetate metabolism hence growth were almost nil.

Batch cultivation. Ethanol inhibition in batch cultures was determined by measuring the specific growth rate of the micro-organism in shake flasks containing P-I medium and 


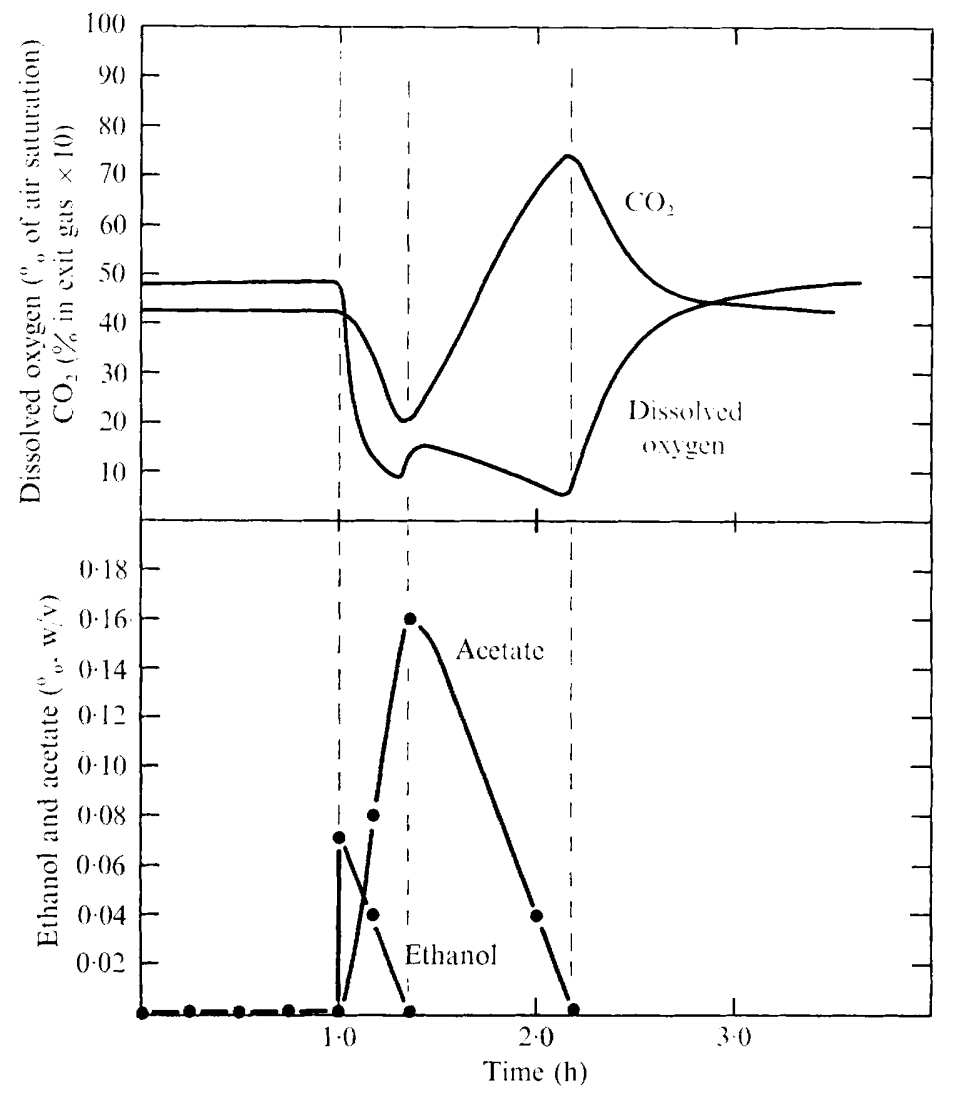

Fig. I. Effect of adding I.48 g ethanol to a 21 ethanol-limited steady state chemostat culture of Acinetobacter calcoaceticus.

various concentrations of ethanol. The maximum specific growth rate was $0.7 \mathrm{~h}^{-1}$ and only at ethanol concentrations above $\mathrm{I} \cdot \mathrm{I} \%(\mathrm{~W} / \mathrm{v})$ was a reduction in growth rate observed (Fig. 2$)$.

Growth inhibition by acetate was measured in batch culture by growing Acinetobacter calcoaceticus on acetate as a sole carbon source. Growth was not inhibited by acetate concentrations of $0.2 \%(\mathrm{w} / \mathrm{v})$ or less (Fig. 3). Since the amount of acetate accumulated in continuous culture (Fig. I) was only $0 \cdot 16 \%(\mathrm{w} / \mathrm{v})$ the acetate concentration per se did not appear to be responsible for inhibiting growth. This was confirmed by adding acetate, to obtain a concentration of $0.2 \%(\mathrm{w} / \mathrm{v})$, to ethanol-limited steady state cultures; such an addition did not inhibit growth, and the acetate was consumed, causing a temporary increase in $\mathrm{CO}_{2}$ evolution.

Regulation of ethanol metabolism. In the experiment depicted in Fig. I, the specific rate of ethanol oxidation before the addition of ethanol was $0.53 \mathrm{~g}$ ethanol $/ \mathrm{g}$ biomass $/ \mathrm{h}$. From this oxidation rate and the corresponding specific growth rate $\left(0.33 \mathrm{~h}^{-1}\right)$, the rate of ethanol oxidation at the maximum specific growth rate $\left(0.7 \mathrm{~h}^{-1}\right)$ was calculated: $0.7 / 0.33(0.53)=$ $\mathrm{I} \cdot \mathrm{I} 2 \mathrm{~g}$ ethanol oxidized $/ \mathrm{g}$ biomass $/ \mathrm{h}$. The specific rate of ethanol oxidation calculated from the slope of the curve of acetate accumulation in Fig. I was $\mathrm{I} \cdot \mathrm{I} 6 \mathrm{~g}$ ethanol oxidized $/ \mathrm{g}$ biomass/h. Thus, the rate of ethanol oxidation (or acetate formation) after the addition of ethanol to the chemostat culture was capable of supporting the maximum specific growth rate although the bacteria were growing at only $0.33 \mathrm{~h}^{-1}\left(47 \% \mu_{\max }\right)$ immediately before 


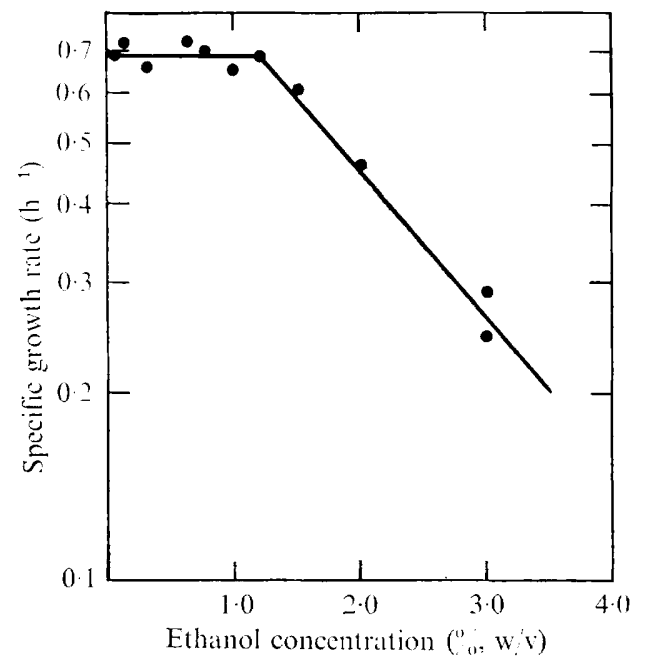

Fig. 2

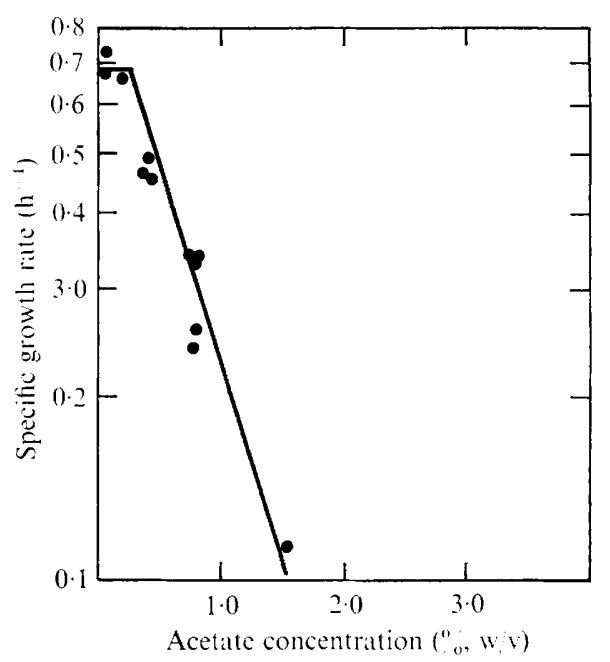

Fig. 3

Fig. 2. Effect of ethanol concentration on the specific growth rate of Acinetobacter calcoaceticus growing on ethanol as a sole carbon source.

Fig. 3. Effect of acetate concentration on the specific growth rate of Acinetobacter calcoaceticus growing on acetate as a sole carbon source.

adding ethanol. Similar high specific rates of acetate synthesis were observed when larger quantities of ethanol were added or when the growth rate and bacterium concentration differed from those in Fig. I.

In another experiment ethanol-grown bacteria were transferred to a shake flask containing P-I medium with an ethanol-acetate mixture as a substrate. The acetate component of the mixture was preferentially utilized and ethanol consumption did not begin until the acetate was depleted (Fig. 4 ). The data indicated that a brief lag period ( $\approx$ I5 $\mathrm{min}$ ) occurred between the time of acetate depletion and the initiation of ethanol metabolism. When the experiment was repeated with a higher initial biomass concentration, some ethanol consumption occurred immediately. Apparently with a larger inoculum the carry-over of ethanol-oxidizing enzymes in the ethanol-grown cells was sufficient to allow measurable ethanol utilization before these enzymes were inactivated or diluted out by growth of the micro-organism.

\section{DISCUSSION}

The addition of a small quantity of ethanol to ethanol-limited chemostat cultures caused a rapid formation and accumulation of acetate. The rate of such acetate formation was sufficient to support the maximum specific growth rate of the organism; however, the growth rate immediately before adding ethanol was only $47 \%$ of the maximum rate. Acetate accumulated for $\mathrm{I} 8 \mathrm{~min}$ after ethanol was added (Fig. I) during which time all of the substrate in the fermenter was accounted for as ethanol and/or acetate, thus, acetate metabolism (and consequently growth) did not occur, as indicated by the rapid decline in $\mathrm{CO}_{2}$ evolution. Generally, when growth ceases the dissolved oxygen (D.O.) tension in a culture rises. However, after ethanol addition D.O. declined for $18 \mathrm{~min}$, this decline presumably reflecting the high rate of NADH oxidation, via cytochrome oxidase, that must occur to regenerate the NAD needed for further ethanol oxidation. 


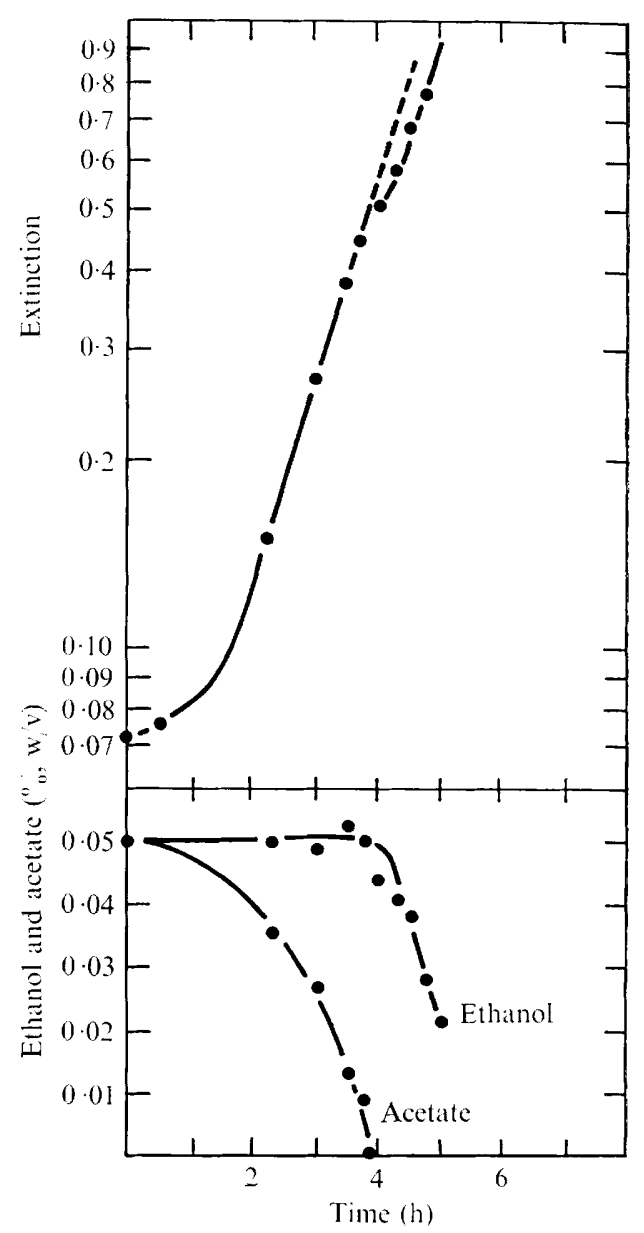

Fig. 4. Growth of Acinetobacter calcoaceticus in a shake flask containing P-I medium and an acetate-ethanol mixture as the sole carbon source. Ethanol and acetate were initially present in $0.05 \%(w / v)$ concentration.

The addition of ethanol increased acetate formation either by inducing the formation of more ethanol dehydrogenase (EDH) and acetaldehyde dehydrogenase (ADH) or by saturating the preformed enzymes with substrate. The induction of higher amounts of EDH and $\mathrm{ADH}$ does not seem likely because the addition of ethanol caused an immediate high rate of acetate synthesis without a lag period for induction. The absence of a lag period is particularly evident from the response of D.O. tension and $\mathrm{CO}_{2}$ evolution, both of which declined noticeably within 2 to 4 min after ethanol addition. Considering the time delay for instrument response, these changes were essentially instantaneous.

In batch culture the acetate component of an ethanol-acetate mixture was preferentially consumed and ethanol utilization did not begin until acetate was depleted. Acetate can prevent ethanol utilization by either feedback inhibition or by repression of enzyme synthesis and these two mechanisms can often be distinguished on a temporal basis. Feedback inhibition immediately prevents enzyme activity in the presence of sufficient concentration of the effecter, but enzyme activity does not immediately cease when a repression control 
mechanism is activated because while the effecter quickly stops enzyme synthesis the activity of the preformed enzyme persists until it decays or is diluted by cell growth. Acetate repression of ethanol metaboiism may account for the effect observed in both batch and chemostat cultures. In the chemostat the accumulation of acetate, while immediately repressing further enzyme synthesis, allows the preformed enzymes to continue synthesizing acetate until ethanol is depleted. In batch culture, when low initial biomass concentrations are used, acetate also prevents ethanol metabolism via repression, but because of the extremely low biomass concentration very little ethanol is oxidized to acetate by the preformed enzymes before enzyme activity decays or is diluted out by growth.

The inhibition of growth that followed the addition of ethanol to ethanol-limited chemostat cultures occurred only during the $18 \mathrm{~min}$ period of acetate accumulation. Neither the amount of ethanol added nor the amount of acetate accumulated inhibited growth in batch culture. The ability of the micro-organism to grow rapidly in an ethanol-acetate mixture (Fig. 4) and the absence of growth inhibition when acetate was added to ethanol-limited cultures indicates that growth inhibition also was not the result of a synergistic effect of ethanol and acetate. Thus, it appears that growth inhibition in the chemostat was not caused by the concentrations of ethanol and acetate per se but was associated in some way with the rapid interconversion of one to the other.

The qualitative aspects of the responses of ethanol-limited cultures to the addition of ethanol have also been observed in chemostat cultures of a thermophilic bacillus (A. Clamen, unpublished data). Thus, the phenomenon does not appear to be unique to the strain of micro-organism used in this study.

The author gratefully acknowledges Dr A. Laskin for classifying the organism used in these studies and Mr C. McCoy for excellent technical assistance.

\section{REFERENCES}

AIBA, S. \& SHODA, S. (I969). Reassessment of the product inhibition in alcohol fermentation. Journal of Fermentation Technology 47, 790-794.

Aiba, S., Shoda, M. \& Nagatani, M. (1968). Kinetics of product inhibition in alcohol fermentation. Biotechnology and Bioengineering 1o, 845-864.

Egamberdiev, N. B. \& Jerusalimskir, N. D. (I968). Effect of ethanol concentration on rate of growth of Saccharomyces vini (RACE Pv-I). Microbiologiya 37, 686-69o.

Hinshelwood, C. N. (1952). The Chemical Kinetics of the Bacterial Cell. Oxford: Clarendon Press.

Holzberg, I., FinN, R. K. \& Steinkraus, K. H. (1967). A kinetic study of the alcoholic fermentation of grape juice. Biotechnology and Bioengineering 9, 413-429.

ThoRnLey, M. J. (1967). A taxonomic study of Acinetobacter and related genera. Journal of General Microbiology 49, 2 I I $1-257$.

ZINES, D. O. \& Rogers, P. L. (1970). The effect of ethanol on continuous culture stability. Biotechnology and Bioengineering I2, 561-575.

ZINES, D. O. \& Rogers, P. L. (I97I). A chemostat study of ethanol inhibition. Biotechnology and Bioengineering $\mathbf{1 3}, 293-308$. 